\title{
Lining material tests for the AUGER PROJECT surface detector
}

\author{
C.O.Escobar ${ }^{a}$, A.C.Fauth ${ }^{a}$, M.M.Guzzo ${ }^{a}$, E.H.Shibuya ${ }^{a}$ \\ ${ }^{a}$ Instituto de Física Gleb Wataghin-UNICAMP, 13083-970 Campinas, São Paulo, Brasil
}

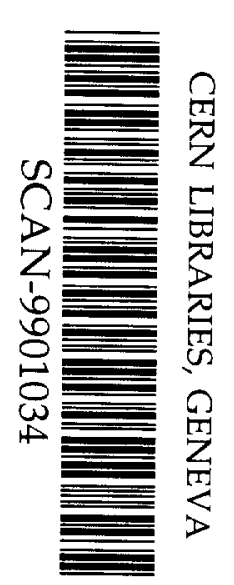

We are trying to obtain a suitable material to compose the lining of a water Cerenkov tank for the surface detector, part of a hybrid detector of the Auger Project. Results of tests were compared with DuPont 1073Tyvek ${ }^{T M}$ and obtained a reasonable performance for $\left(\mathrm{PVC}+\mathrm{BaSO}_{4}\right)$ material.

\section{Introduction}

A hybrid detector proposed to the Pierre Auger Project is an array with water Cerenkov tanks and mirrors to observe fluorescence light in the atmosphere, both produced by particles of Cosmic Radiation entering in the atmosphere and multiplicating through. The main aim of the Pierre Auger Project is to detect Extensive Air Shower with energy $\mathrm{E}>10^{19} \mathrm{eV}$ and particular interest to increase the statistics for $\mathrm{E} \sim 10^{20} \mathrm{eV}$, where GZK cut-off could exist.

Our contribution to the engineering phase of the Pierre Auger Project is restricted to a search for a convenient reflective material. For that purpose we measured the reflection of some commercially available materials and a specially made $\mathrm{PVC}+\mathrm{BaSO}_{4}$ material, under request and recipes to SANSUY S/A - INI. DE PLÁSTICOS, a Brazilian plastic industry.

First, we did measurements of relative diffuse reflection on dry materials, using a PERKINELMER, Lambda 9 - series 1645 - UV/VIS/NIR Spectrometer, part of IFGW/UNICAMP facilities. In a second step, a flexible tank full of tap water, lined by $\left(\mathrm{PVC}+\mathrm{BaSO}_{4}\right)$ or DuPont 1073Tyvek was used. Also measurements of transmitance was done for both materials and we found that no transmitted signal was observed for the PVC bag, then this one is sufficiently shielded against external light effect on the Cerenkov light produced inside the tank.

\section{Measurements}

Figures 1 shows the beforehand mentioned measurements of dry materials. As the Cerenkov light produced inside the tank by Cosmic-Ray particles is in a ultraviolet region, we disregard several other tested materials, for instance $\mathrm{TiO}_{2}$. Some other tested material, mainly thermoluminescent materials (Teflon $+\mathrm{BaSO}_{4}$ : Eu or Teflon $+\mathrm{Mg}_{2} \mathrm{SiO}_{4}$ ) showed relative diffuse reflection greater than $100 \%$. This could be caused by $\mathrm{Eu}$, that is the essential luminescent chemical element, taking into account that the reference material of the spectrophotometer for the diffuse relative reflection measurements is an $\mathrm{Al}$ plate painted with $\mathrm{BaSO}_{4}$, as is written in the spectrometer's manual. From these results and observations we decided to concentrate the measurements in the $\left(\mathrm{PVC}+\mathrm{BaSO}_{4}\right)$ material, testing many combinations of chemical elements concentrations and thickness of the sheets.

Afterwards we measured the signal of cosmicray particles (mainly muons) in a water tank, first with $\left(\mathrm{PVC}+\mathrm{BaSO}_{4}\right)$ and after with DuPont 1073 Tyvek bags, working as lining material. The tank has diameter $=1.2 \mathrm{~m}$, height $=1.3 \mathrm{~m}$, filled with $\approx 1,500$ liters of tap water and provided with a photomultiplier Phillips XP2040 optically coupled to a plexiglas base. A pair of plastic scintillators, with different sizes was located over the tank. Following preliminary observations, calibrations and measurements, we moved one of the plastic scintillators to be under the water tank, in a vertical line linking the upper scintillator, the tank and the lower (smaller) scintillator, ex- 
pecting to discriminate only the vertical muons measurements.

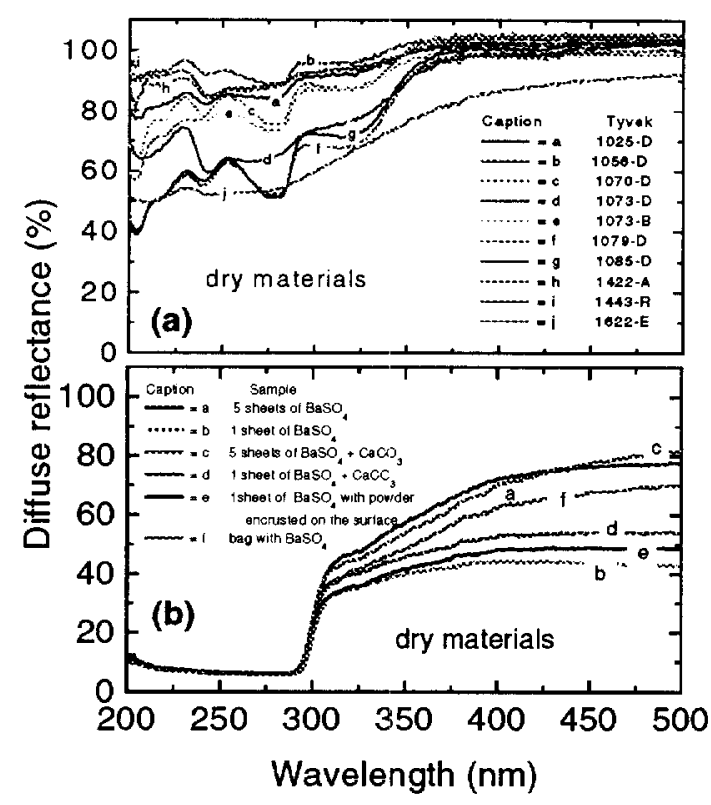

Figure 1. Diffuse reflectance of dry materials

Defining the ratio between the 3 -fold ( 2 plastic scintillators + water tank) and the 2 -fold (2 plastic scintillators) coincidences countings as a quantity proportional to an counting efficiency of the water Cerenkov tank, we got the the set of Figures 2 (the signal threshold was $20 \mathrm{mV} / 50 \Omega$ ).

\section{Conclusions and Discussions}

Analysis of $\left(\mathrm{PVC}+\mathrm{BaSO}_{4}\right)$ combination shows a little less efficiency than DuPont Tyvek1073, as shown in Figures 1 and 2. But in this material the decrease of signal is more pronounced than in $\left(\mathrm{PVC}+\mathrm{BaSO}_{4}\right)$. This effect in Tyvek was already reported by Bai Xinhua, using an American Chinese tank. His explanation of this effect is related

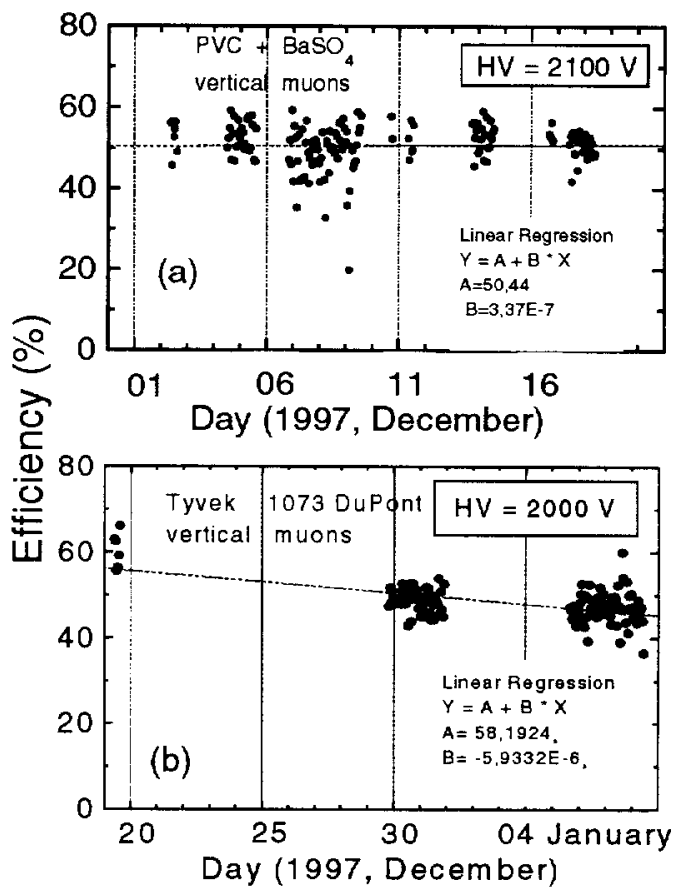

Figure 2. Efficiency estimation

to lower Tyvek reflectivity caused by water filling of Tyvek fibers or by the activity of remnant bacteria in the water.

The Cerenkov light detected by the photomultiplier is a superposition of the totally reflected light by the lining material. If so, when the reflective index of the material changes with time the Cerenkov signal also should change. The $\left(\mathrm{PVC}+\mathrm{BaSO}_{4}\right)$ material is compact, whereas the Tyvek have air gaps, because it is made with fibers encrusted in a polyethylene sheet. So, the total reflection of Cerenkov light in Tyvek could suffer decrease as time of soaking goes, due to the progressively occupation of air gaps by more reflective material(water and/or fibers composing the material)

To look for a possible effect of soaking that may 
explain the observed decrease of signal, we tested small samples of DuPont 1073Tyvek utilized inside the tank, a ( $\left.\mathrm{PVC}+\mathrm{BaSO}_{4}\right)$ sheet also utilized inside the tank and a (Tyvek+black polyethylene sheet welded together) made and utilized at Fermilab measurements. Results from these measurements are not conclusive about the effect, in spite of small decrease of signal along the time of soaking, effect that is not observed in the $\left(\mathrm{PVC}_{+} \mathrm{BaSO}_{4}\right)$ material probably because this material is more compact (Figures 3 ).

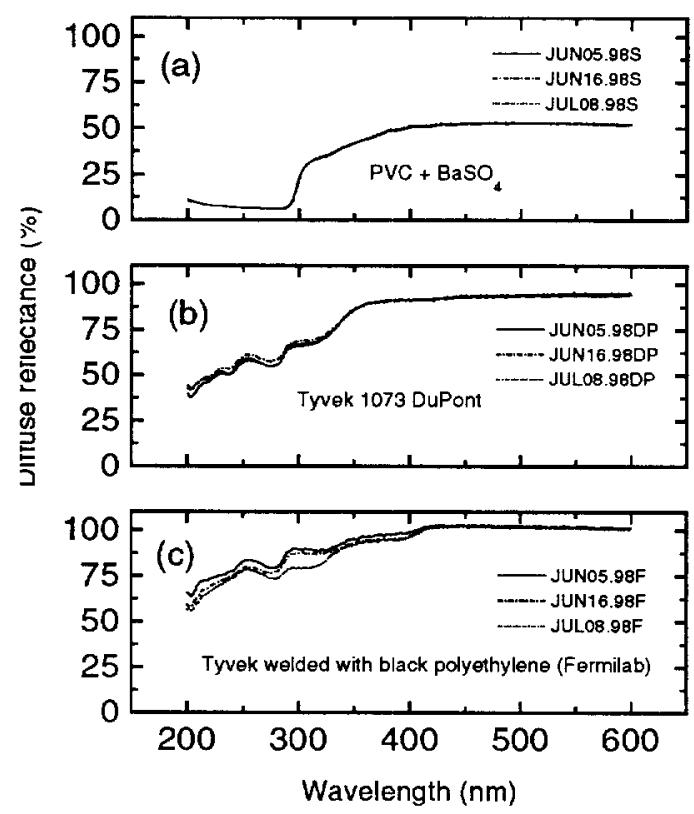

Figure 3. Diffuse reflectance measurements parametrized by time of soaking

Apparently, the wetting effect is more pronounced in the (Tyvek+black polyehylene) than in our previously wetted DuPont Tyvek1073. In any way the effect of soaking is not conclusive, but it is necessary to remark that the wetting conditions of the Tyvek inside the tank and the series of tests shown in Figures 3 are different, because these tests was done with the sample materials embedded with deionized water in a small cups of $1 \mathrm{ml}$ capacity. Then all observations could be consistent with a possible effect of soaking in Tyvek and so the Sansuy made $\left(\mathrm{PVC}+\mathrm{BaSO}_{4}\right)$ combination could be a reasonable alternative for the lining of the tank because it doesn't have pronounced air gaps that could be filled with water and bacteria.

\section{Aknowledgments}

The authors would like to thanks many people for the support and advices, specially to the Brazilian branch of DuPont (Mr. O.Porto) and SANSUY S/A (T.Honda, Y.Hirasaki, S.Morita, R.O.Brostel, H.Imagawa, A.C.Iguchi, G.Hanashiro) for the financial support and/or free of charge of materials and technical services. Also we are indebted to Mr.A.C.da Costa for the help and measurements of reflectivity and transmittance on dried materials. We had also a financial support from CPG-IFGW/UNICAMP for materials. One of us (EHS) acknowledgs to FAEP/UNICAMP and to the Organizing Committee of $\mathrm{X}^{t h}$ ISVHECRI (Prof.O.Saavedra) for the support to present this report at the Symposium and to Prof.F.Galembeck for many advices and informations about reflectivity of materials. Finally we are grateful to Prof. R.C.Shellard, who first called our attention to the possibility of a flexible plastic tank use. 